\title{
Compensated Diffusion Magnetic Resonance Imaging
}

Edward Li

Mohammad Javad Shafiee

Ameneh Boroomand

Farzad Khalvati

Masoom A. Haider

Alexander Wong
University of Waterloo, ON, Canada

University of Waterloo, ON, Canada

University of Waterloo, ON, Canada

University of Toronto, ON, Canada

University of Toronto, ON, Canada

University of Waterloo, ON, Canada

\section{Abstract}

This paper presents a novel compensated diffusion magnetic resonance imaging (cdMRI) system for improved tissue detail and contrast for screening and diagnosis. The proposed cdMRI system incorporates the intrinsic properties of the MRI apparatus to compensate for artifacts and degradation caused through the imaging process. Experimental results for prostate imaging show that significant improvements in tissue detail and contrast can be obtained compared to current MRI systems.

\section{Introduction}

Magnetic Resonance Imaging (MRI) is a crucial medical imaging technology for the screening and diagnosis of different types of diseases. In particular, diffusion MRI (dMRI) can be used to measure the diffusion of water in biological tissue, and has been found to be a powerful tool for tissue differentiation in applications such as cancer imaging. However, due to the intrinsic properties associated with dMRI imaging, degradations and artifacts are introduced in the imaging process that result in reduced tissue detail and contrast in the captured dMRI data. Therefore, a dMRI imaging system that can produce greater tissue detail with higher contrast is highly desired for diagnostic purposes.

Motivated to improve dMRI imaging quality, this paper presents a novel compensated diffusion magnetic resonance imaging (cdMRI) system that takes into account the intrinsic properties of the MRI apparatus to improve tissue detail and contrast for screening and diagnosis. Such a system allows for the acquisition of dMRI imaging data that can provide radiologists with more detailed information to aid in clinical decision support.

\section{Methodology}

The proposed dMRI system can be summarized as follows. First, a calibration process is performed to quantitatively characterize the intrinsic properties of the MRI apparatus in the form of a compensation function $C$. The measured signal $X$, along with $C$, are then used to form a cdMRI image $Y$.

The reconstruction framework used in this paper extends upon the reconstruction framework proposed in [1], where the $k$-space measurements $X$, and data- and spatial-driven constraints are used in addition to newly proposed compensation function $C$ to form cdMRI image $Y$. More specifically, we treat the problem of forming $Y$ as a Maximum a Posteriori (MAP) problem, where we maximize the following probability $P(Y \mid X)$ using an extended CD-SFCRF formulation:

$$
P(Y \mid X)=\frac{1}{Z(X)} \exp (-\psi(Y \mid X))
$$

$Z(X)$ is the normalization function and $\psi($.$) is some combination$ of unary and pairwise potential functions, denoted by $\psi_{u}$ and $\psi_{p}$, respectively:

$$
\psi(Y \mid X)=\sum_{i=1}^{n} \psi_{u}\left(y_{i}, X\right)+\sum_{\varphi \in C} \psi_{p}\left(y_{\varphi}, X\right)
$$

The unary potential enforces original $k$-space measurements as well as accounting for the compensation function $C$ which compensates for the degradation function $F$, while the pairwise potential enforces data and spatial consistencies. The unary and pairwise potential functions, $\psi_{u}$ and $\psi_{p}$, are formulated in Eq 3 and 4, respectively:

$$
\begin{aligned}
\psi_{u}\left(y_{i}, X\right) & =x_{i}-F\left(C\left(y_{i}\right)\right) \\
\psi_{p}\left(y_{i}, y_{j}, X\right) & =e^{\left(-\frac{\left(x_{i}-x_{j}\right)^{2}}{\sigma}\right)}\left(F\left(x_{i}\right)-F\left(x_{j}\right)\right)
\end{aligned}
$$

Fig. 1 illustrates a graphical representation for the random field model used for cdMRI formation.

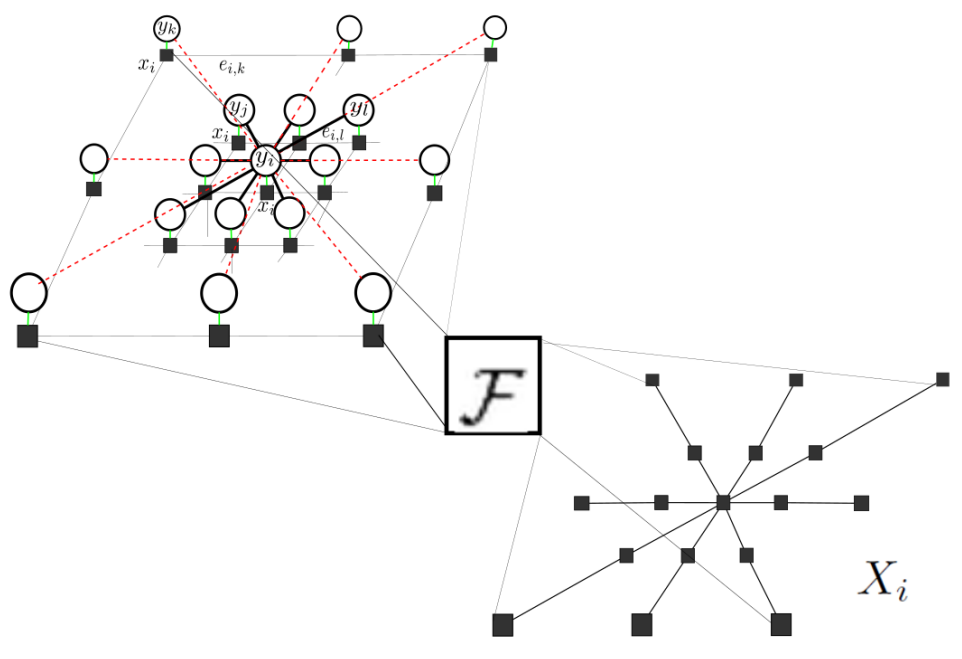

Fig. 1: Random field model used for cdMRI formation

\section{Results and Discussion}

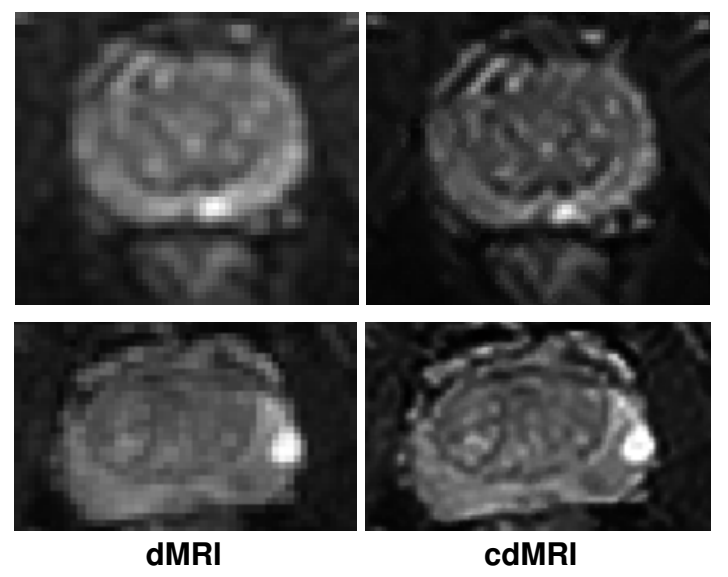

Fig. 2: Comparison between prostate dMRI imagery obtained using standard dMRI (left) and cdMRI (right)

Fig. 2 shows a comparison between prostate dMRI imagery obtained using standard dMRI and cdMRI. Visually, it can be observed that the proposed cdMRI system was able to produce dMRI imagery with significantly improved tissue detail and contrast compared to standard dMRI, which is very useful for providing more information for diagnostic purposes. Future work involves adapting the cdMRI methodology for improving other MRI modalities such as CDI [2], High-b DWI [3], T1w, and T2w imaging.

\section{References}

[1] Li, E.,Shafiee, M. J., Chung, A. , Khalvati, F., Wong, A. and Haider, M. Enhanced Reconstruction of Compressive Sensing MRI via Cross-Domain Stochastically Fully-Connected Random Field Model ISMRM 2015 (2015).

[2] Wong, A., Glaister, J., Cameron, A., and Haider, M. Correlated Diffusion Imaging. BMC Medical Imaging (2013).

[3] Shafiee MJ, Haider SA, Wong A, Lui D, Cameron A, Modhafar A, Fieguth P, Haider MA. Apparent Ultra-High b-Value Diffusion-Weighted Image Reconstruction via Hidden Conditional Random Fields. IEEE TMI (2015). 